\title{
A Novel Device for Passive Restraint of the Tongue as an Adjunct to Mandibular Advancement Therapy in Incomplete Responders
}

\author{
Gregory Turek, BDS (Syd)
}

Somcentre Dental Sleep Medicine Clinic, Sydney, NSW Australia

\begin{abstract}
Tongue restraint may provide additional benefit in mandibular advancement splint (MAS) therapy. However, previous methods of tongue restraint are based on applying active force, resulting in a substantial degree of discomfort for most users. This report describes a novel method called Tongue Tamer (TT), which uses the natural passive 'flow' of the tongue to fill the space available to it when relaxed. The TT has been added to the lower plate of patients with an incomplete response to MAS therapy. In a series of 17 cases, the addition of TT has resulted in an additional reduction of $-41.8 \pm 67.3 \%$ (mean \pm standard deviation) in apnea-hypopnea Index (AHI) compared to MAS therapy alone and $30 \%$ of patients were converted to complete responders (AHI $<5$ events/h). This novel method of passive restraint of the tongue may be a useful adjunct to MAS therapy.
\end{abstract}

Keywords: passive tongue restraint; adjunct to MAS therapy; oral appliance therapy; OSA

Citation: Turek, G. A novel device for passive restraint of the tongue as an adjunct to mandibular advancement therapy in incomplete responders. J Dent Sleep Med. 2019;6(2)

\section{INTRODUCTION}

Mandibular advancement splint (MAS) therapy does not provide adequate reduction of the apnea-hypopnea index (AHI) in 36\% of patients with obstructive sleep apnea (OSA). ${ }^{1}$ The addition of a tongue retainer was previously reported as a potential method to enhance MAS efficacy. ${ }^{2}$ Although tongue restraint would likely be helpful in reducing OSA in many patients, previous methods have relied on active force such as gripping, pressing, sucking, or electrical stimulation. Active tongue restraint via a suction bulb does reduce AHI; however, it is associated with significant discomfort and subsequently poor device usage. ${ }^{3}$

This report describes a novel concept of tongue stabilization through passive means. It aims to use natural passive 'flow' of the tongue to fill any surface or shape on which it is placed when muscle tone is low, such as during sleep. The device, called the Tongue Tamer (TT), is a uniquely shaped set of thin wire frames attached to the lower splint to sit on either side of the tongue (Figure 1). The speculation is that the relaxed tongue, with very low muscle tone, would envelop the spaces created by the TT, which would thereby act as a restraint for the tongue. The theory is that this type of tongue restraint would be relatively imperceptible to the wearer in comparison with active methods previously investigated. The TT was added to the MAS device of a series of patients with clinical OSA who had an inadequate response to MAS therapy.

\section{METHODS}

All cases were selected to trial the TT addition to their MAS device if a sleep study with standard MAS indicated an inadequate response to therapy. All cases were referred by sleep specialists to the Somcentre Dental Sleep Medicine Clinic (Sydney, Australia) for treatment of OSA with standard MAS therapy (Somnodent). The response to standard MAS therapy was confirmed by a follow-up sleep performed at the request of the referring physician at accredited sleep laboratories in the Sydney area (following a settling-in period and optimal titration). In all presented cases, the sleep study with standard MAS therapy indicated an AHI greater than 5 events per hour, indicating OSA was still present with MAS therapy. A TT was constructed using two frames in an elongated loop shape to sit along either side of the tongue. To construct the TT loops, wire of two different gauges have been tested (half round $2 \mathrm{~mm}$ in width or round $0.8 \mathrm{~mm}$ ). A TT structure has also been made of plastic loops for the purpose of imaging but has not been tested in terms of outcomes. The TT structure was added to the existing MAS device (at the same titration setting) and a further follow-up sleep study was arranged. Sleep studies were conducted as part of clinical care at different centers; as such the sleep recording or reporting was not standardized between specialist sleep centers. However, in all cases both sleep studies were conducted at the same center and by the same method. The AHI was compared between the sleep study on standard MAS therapy and the sleep study with MAS modified with the TT structure. The percent change in AHI between the two studies was calculated. Data were extracted from available 
copies of sleep study reports or follow-up letters from referring physicians.

Figure 1. The Tongue Tamer device on the lower plate of a mandibular advancement splint.
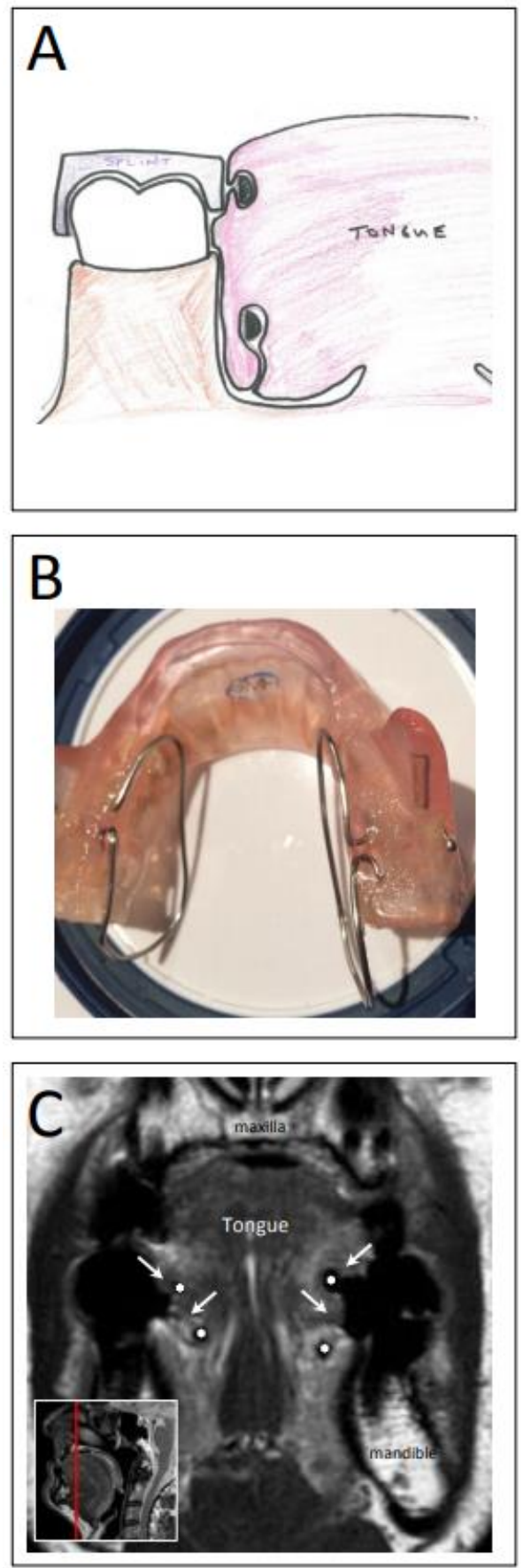

(A) Schematic illustration of the Tongue Tamer (TT) concept of retention of the tongue. When relaxed the tongue tissue fills the spaces created by the TT structure. (B) The TT structure (wire frames) affixed to the lower plate. (C) Magnetic resonance imaging (MRI) scan of the MAS with TT structure added (constructed of plastic material to allow imaging) from a user of the device. A coronal image shows the cross-section of the TT structure $\left({ }^{*}\right)$. The soft tissue of the tongue can be seen enveloping the TT frame (arrows). Inset: sagittal view showing location of the coronal slice (red line).

\section{RESULTS}

Data from 17 individuals are reported in Table 1. All sleep study data are reported as AHI for total sleep time, which was available for all subjects. Where available, AHI from a diagnostic study has been included in the table. Thirteen of these 17 cases appear to have substantially reduced their AHI with the TT modification compared to MAS therapy alone (based on a minimum $30 \%$ reduction in $\mathrm{AHI})$. In some of these cases (B, D, J, Q) the improvement has resulted in a complete response, or cure of OSA.

In terms of user perceptions of the TT device, no formal analysis has been performed. However, as part of follow-up, patients were questioned about the comfort and experience of wearing the TT device. Most patients commented that they could not feel the TT addition or it was minimally perceptible. In some cases, the TT frames did cause initial discomfort because of too much posterior extension or extension too deep into the lingual sulcus, which required the TT frames to be remoulded to reduce this.

\section{DISCUSSION}

The TT is a novel method that may restrain the tongue. The TT has been applied to a number of MAS devices of patients with clinical OSA with an inadequate therapeutic response. Repeat sleep studies with the addition of the TT have shown significant improvement in most cases and dramatic improvement in some. Incomplete response to MAS therapy is an important issue, particularly in patients in whom CPAP has failed. Adaptions to improve the outcome of MAS therapy are therefore of great interest and the tongue is an ideal target to potentially enhance therapeutic effect. A previous study ${ }^{2}$ has illustrated the benefit of combining MAS and tongue restraint (via suction bulb). However, this study also emphasized discomfort and potential poor compliance with using a tongue suction bulb. The TT concept is a completely different take on tongue restraint as it aims to accomplish this through a passive method in that the tongue naturally envelops the provided spaces, rather than using forces such as suction to protrude it. The reason for desiring such a method is that discomfort is associated with trying to actively hold the tongue in the oral cavity, as seen with suction bulbs. ${ }^{3}$ Although this study cannot confirm whether the tongue was actually restrained by the TT device during the sleep studies, imaging of a TT-modified MAS in a single user illustrates the tongue tissue infiltrating the space created by the TT structure. The additional AHI improvement observed in most presented cases suggests the TT modification is providing benefit in addition to MAS therapy alone. The TT is a different concept of tongue restraint compared to previous tongue suction devices (targeting the tongue base compared to the tongue 
tip). It would be interesting to compare the treatment outcomes of these two devices in future studies. Of course, there are individual differences in response to the TT device; however, it is unclear at this stage which patient types may particularly benefit from this type of oral therapy. Phenotypic factors such as oropharyngeal crowding, supine dependency, and sex may prove to identify suitable candidates. Anecdotally, a particularly good response has been observed in males with a crowded oropharynx (or 'big fat tongue'). Patients with OSA for whom oropharyngeal collapse due to a large tongue is not the primary cause of OSA may therefore not receive benefit from a TT device, but this requires formal investigation.

\section{CONCLUSION}

Preliminary evidence suggests using the novel TT device may provide additional benefit for those in whom MAS treatment has failed. For the first time, this method describes the potential for tongue restraint in a way that does not require active methods such as suction and that appears to be comfortably worn by patients.

\section{ACKNOWLEDGMENT}

The author would like to thank Kate Sutherland for her assistance in review of the manuscript.

\section{REFERENCES}

1. Sutherland K, Takaya H, Qian J, Petocz P, Ng AT, Cistulli PA. Oral appliance treatment response and polysomnographic phenotypes of obstructive sleep apnea. J Clin Sleep Med. 2015;11(8):861-868.

2. Dort L, Remmers J. A combination appliance for obstructive sleep apnea: the effectiveness of mandibular advancement and tongue retention. J Clin Sleep Med. 2012;8(3):265-269.

3. Deane SA, Cistulli PA, Ng AT, Zeng B, Petocz P, Darendeliler MA. Comparison of mandibular advancement splint and tongue stabilizing device in obstructive sleep apnea: a randomized controlled trial. Sleep. 2009;32(5):648-653.

\section{SUBMISSION \& CORRESPONDENCE INFORMATION}

\section{Submitted for publication June 9, 2018}

Submitted in final revised form October 7, 2018

Accepted for publication November 25, 2018

Address correspondence to: Dr. Gregory Turek, BDS, Somcentre Dental Sleep Medicine Clinic, Level 3, 20 Clarke St., Crows Nest, NSW 2065 Australia, Tel: +61-29467-0400, Fax: +61-2-9467-0467, Email: gregoryturek@optusnet.com.au

\section{DISCLOSURE STATEMENT}

The author is the inventor of and holds the patent for the Tongue Tamer method. 
Table 1. Sleep study data in series of patients with clinical OSA initially treated with a standard MAS appliance and then with addition of the novel Tongue Tamer modification.

\begin{tabular}{|c|c|c|c|c|c|c|c|c|c|c|c|}
\hline Case & Sex & Age & BMI & $\begin{array}{l}\text { Diagnostic } \\
\text { study year }\end{array}$ & $\begin{array}{c}\text { Diagnostic } \\
\text { AHI }\end{array}$ & $\begin{array}{l}\text { Overnight sleep } \\
\text { assessment } \\
\text { method^ }^{\wedge}\end{array}$ & $\begin{array}{c}\text { MAS study } \\
\text { year }\end{array}$ & $\begin{array}{l}\text { MAS+TT } \\
\text { study year }\end{array}$ & MAS AHI & $\begin{array}{c}\text { MAS+TT } \\
\text { AHI }\end{array}$ & $\begin{array}{c}\% \text { change AHI with } \\
\text { addition of TT vs MAS } \\
\text { alone }\end{array}$ \\
\hline $\mathbf{A}$ & Male & 56 & 25.5 & 2008 & 66.7 & PSG & 2014 & 2016 & 9 & 6 & -33.3 \\
\hline B & Male & 71 & 26.1 & unk & unk & PSG & 2014 & 2016 & 17.7 & 0.3 & -98.3 \\
\hline C & Male & 37 & 26.3 & unk & unk & HST & 2012 & 2012 & 48 & 11.3 & -76.5 \\
\hline D & Male & 69 & 27.2 & 2013 & 32.3 & PSG & 2011 & 2013 & 10.5 & 0 & -100.0 \\
\hline $\mathbf{E}$ & Male & 71 & 27.7 & unk & unk & PSG & 2014 & 2015 & 15.2 & 5.7 & -62.5 \\
\hline $\mathbf{F}$ & Male & 70 & 29.4 & 2008 & 16.1 & PSG & 2012 & 2014 & 16.8 & 9 & -46.4 \\
\hline $\mathbf{G}$ & Male & 56 & 30.5 & unk & unk & HST & 2011 & 2011 & 46.8 & 4 & -91.5 \\
\hline $\mathrm{H}$ & Male & 59 & 30.7 & unk & unk & HST & 2011 & 2012 & 26.9 & 11.2 & -58.4 \\
\hline I & Male & 66 & 32.7 & 2012 & 19.7 & PSG & 2014 & 2015 & 17.2 & 22.3 & +29.7 \\
\hline $\mathbf{J}$ & Male & 39 & 36.1 & 2011 & 33.7 & PSG & 2012 & 2013 & 23 & 0.9 & -96.1 \\
\hline $\mathrm{K}$ & Male & 49 & 37.8 & 2013 & 25 & PSG & 2014 & 2015 & 22 & 13.5 & -38.6 \\
\hline $\mathbf{L}$ & Male & 60 & 53.6 & unk & unk & HST & 2011 & 2012 & 68.6 & 36.5 & -46.8 \\
\hline $\mathbf{M}$ & Male & 64 & & 2013 & & PSG & 2014 & 2015 & 25 & 14 & -44 \\
\hline $\mathbf{N}$ & Female & 69 & 25.8 & 2010 & 57 & PSG & 2012 & 2013 & 19.5 & 55.1 & +182.6 \\
\hline 0 & Female & 71 & 29.1 & unk & unk & PSG & 2012 & 2015 & 22.5 & 17.5 & -22.2 \\
\hline $\mathbf{P}$ & Female & 47 & 29.7 & 2011 & 15.4 & HST & 2012 & 2012 & 6.8 & 5.5 & -19.1 \\
\hline $\mathbf{Q}$ & Female & 60 & 31.5 & unk & unk & PSG & 2015 & 2015 & 16.4 & 1.7 & -89.6 \\
\hline
\end{tabular}

SD)

$\mathrm{AHI}=$ apnea-hypopnea Index, HST = home sleep test, MAS =mandibular advancement splint, PSG = in-laboratory polysomnography, TT = Tongue Tamer, unk = unknown information

*Although sleep assessment method varies between patients, in all cases the MAS and MAS-TT study were both conducted at the same center and by the same method 